\title{
Electrocardiogram Evaluator
}

National Cancer Institute

\section{Source}

National Cancer Institute. Electrocardiogram Evaluator. NCI Thesaurus. Code C83259.

A person who determines the significance of an electrocardiogram assessment. 